Volumen 24, No 1, Páginas 17-24

IDESIA (Chile) Enero - Abril 2006

\title{
EVALUACIÓN TÉCNICO-ECONÓMICA DE LA PRODUCCIÓN DE CALAS DE COLOR EN LA REGIÓN DE LA ARAUCANÍA, CHILE
}

\author{
TECHNICAL AND ECONOMIC EVALUATION OF THE PRODUCTION OF \\ COLOURED CALLA LILIES IN THE ARAUCANÍA REGION, CHILE
}

\author{
Berta Schnettler Morales ${ }^{1}$; Armando Mera Sáez ${ }^{1}$; Rodolfo Pihán Soriano ${ }^{1}$
}

\begin{abstract}
RESUMEN
Con el propósito de buscar nuevas alternativas productivas para la IX Región de La Araucanía, caracterizada por el desarrollo de rubros agrícolas tradicionales, se estudió la factibilidad técnica y económica de comercializar calas de color en florerías de Santiago y principales ciudades del sur de Chile. Se simuló la implementación de una unidad productiva de calas en la comuna de Lautaro, utilizando túberos como material de propagación. La inversión inicial correspondió a M\$ 86.924 con un capital de trabajo de M\$16.949, considerando una producción en régimen de 57.904 varas de cala, de la cual el 80\% corresponde a primera selección y el resto a segunda. Se obtuvo un valor actual neto (VAN 12\%) de M\$ -70.138 y una tasa interna de retorno modificada (TIRM) de 3,1\% para el proyecto financiado íntegramente con recursos propios y un VAN $(9,3 \%)$ de M\$ -41.472 y una TIRM negativa al considerar un aporte propio de 50\% y el resto financiado vía deuda. La pérdida obtenida se explica por el elevado valor de inversión inicial y reinversiones necesarias en material vegetal de propagación y por los bajos precios pagados por la producción de varas de segunda calidad.
\end{abstract}

Palabras clave: Calas de color, rentabilidad, valor actual neto.

\begin{abstract}
In the search for new production alternatives for The Araucanía Region of Chile, which is characterized by the development and production for traditional agriculture customs, a study was carried out to examine the technical and economic feasibility of selling coloured calla lilies in flower shops in Santiago and the principal cities of the southern part of the country. A simulation was made of a calla lily production unit in the municipality of Lautaro, using tubers as a propagation material. The initial investment was Ch\$86,924,000 with a working capital of Ch\$16,949,000, and production based on 57,904 stems of calla, 80\% of which would be prime quality and the remainder of which would be seconds. The results obtained a Net Present Value (NPV 12\%) of Ch\$ -70,138,000 and a modified internal rate of return (MIRR) of 3.1\% for the project if financed entirely with its own resources; and a NPV (9.3\%) of Ch\$-41,472,000 with a negative MIRR based on an investment of 50\% of its own resources, with the rest financed by debt. The resulting loss may be explained by the high cost of the initial investment and the reinvestment necessary in plant material for propagation, as well as the low prices paid for stems of second quality.
\end{abstract}

Key words: Coloured calla lilies, profitability, net present value.

\section{INTRODUCCIÓN}

La agricultura de la IX Región de La Araucanía se caracteriza por su alta dependencia respecto de rubros tradicionales, dentro de los cuales destacan los cereales y la ganadería. Su creciente pérdida de competitividad motiva la necesidad de bus- car nuevas alternativas productivas dentro de las cuales se encuentra la floricultura, rubro que se ha expandido tanto territorialmente en el país, como en variedad de especies cultivadas. Dentro de éstas la cala, Zantendeschia K. Spreng es un género cuyo cultivo es relativamente nuevo a nivel mundial, con una expansión en el mercado internacio-

1 Universidad de La Frontera, Facultad de Ciencias Agropecuarias y Forestales, Casilla 54-D, Temuco, Chile. E-mail: bschnett@ufro.cl

Fecha de Recepción: 16 Febrero de 2006

Fecha de Aceptación: 31 Marzo de 2006 
nal para flores de corte y túbero (Funnel et al., 1998; Clemens et al., 1998). La disponibilidad de híbridos con grandes flores de diferentes colores ha hecho que se convierta en una flor de corte muy popular en Nueva Zelandia, Japón y Europa (Tjia, 1987; Kritzinger et al., 1998).

Esta especie es producida por medianos y grandes agricultores de las regiones IX y X del país (ODEPA, 2005), mostrando una buena adaptación a las condiciones edafoclimáticas de esta zona. Sin embargo, este género es muy susceptible a la enfermedad pudrición blanca en los túberos causada por la bacteria Erwinia carotovora, que puede causar pérdidas del $100 \%$ en una plantación (Etcheverría, 2002). Al respecto, la literatura internacional señala que el uso de multiplicación in vitro permite una producción inmediata de plantas homogéneas de cala libre de virosis (Funnel et al., 1988) y se evita el daño producido por Erwinia spp (Cortés-Monllor, 1990).

Las calas representan un $11 \%$ de los envíos nacionales de flores al exterior. Su producción y exportación ha adquirido gran importancia en los últimos dos años. El 61\% de sus exportaciones tiene como destino Holanda, y el 39\% restante, Estados Unidos. Aproximadamente, el 50\% de los envíos se realiza durante el mes de diciembre (ODEPA, 2005).

Respecto al mercado nacional para esta flor, Mera (2004), mediante entrevistas personales a dueños de 119 florerías de Santiago y de las principales ciudades del sur de Chile: Concepción, Temuco, Valdivia, Osorno y Puerto Montt determinó una demanda anual aproximada de 160.000 varas concentradas entre noviembre y febrero de cada temporada, las cuales son adquiridas principalmente por personas de estratos altos y medios.

Considerando estos antecedentes, el presente estudio tuvo como objetivo determinar la rentabilidad de la comercialización de calas de color producidas en la IX Región en el mercado de las principales ciudades del sur de Chile y Santiago, considerando abastecer el $20 \%$ de la demanda con un incremento del $10 \%$ anual.

\section{MATERIALES Y MÉTODOS}

Se eligió la zona de Lautaro, Provincia de Cautín en la IX Región, perteneciente al valle central con suelos de la serie Temuco. En estas condicio- nes edafoclimáticas se simuló la implementación de una unidad productiva de calas de color, ubicada a $20 \mathrm{~km}$ al norte de la ciudad de Temuco, con una superficie de plantación de $1.055 \mathrm{~m}^{2}$ para el primer año, hasta alcanzar $1.550 \mathrm{~m}^{2}$, a partir del quinto año de evaluación. Se utilizó una densidad de plantación de 36 túberos $/ \mathrm{m}^{2}$, con una producción promedio de 1,5 flores por túbero, lo que permite obtener 54 flores $/ \mathrm{m}^{2}$, de las cuales el $70 \%$ tiene valor comercial, correspondiendo el $80 \%$ a flor de primera selección y el resto a segunda selección. Las variedades de calas utilizadas correspondieron a Black Magic y Florex Gold de color amarillo; Hotshot, Sensation y Mango de color naranjo; Magestic Red de color rojo; Lilac Mist de color lila, y Pink Persuasion de color rosado.

La adquisición de los túberos se realizó previo a la puesta en marcha del proyecto como parte de la inversión inicial y se realizaron reinversiones en los años 4 y 7 de la evaluación, considerando un precio de compra de \$1.008/túbero. Se consideró utilizar el material vegetal durante 4 años como máximo con una eliminación anual del $25 \%$ de los túberos por problemas fitosanitarios según lo indicado por Chaín (2000).

Se consideró el establecimiento del cultivo en forma escalonada para abastecer las florerías entre diciembre y abril de cada temporada, con el $30 \%$ de la producción en los meses de diciembre y febrero, $20 \%$ en enero y $10 \%$ en marzo y abril. De esta forma, sobre la base de la tecnología de producción propuesta por Chaín (2000), el cultivo de calas se inicia con la preparación de suelo entre los meses de septiembre a febrero que consiste en la realización de barbecho químico, labores culturales y confección de platabandas. Se contempló riego por cintas y aplicaciones de agroquímicos para control de plagas y enfermedades que afectan el cultivo (Botrytis, Acremonium, Alternaria, Erwinia carotovora, trips, pulgones, babosas y caracoles). Se consideró la cosecha manual de las flores y su clasificación de acuerdo a parámetros de calidad (primera selección: varas de 40 a $50 \mathrm{~cm}$ de largo, segunda selección: 30 a $40 \mathrm{~cm}$ ) en packing, para ser almacenadas posteriormente en cámara de frío hasta el momento de la comercialización en cajas de cartón. Se contempló, además, la cosecha de túberos una vez muerto el follaje en los meses de marzo y abril, los cuales son sometidos a un proceso de curado en cámara para la suberización de la epidermis. 
Los ingresos por venta se calcularon considerando los precios determinados por Mera (2004) para la comercialización de calas de color en las ciudades de Santiago (\$ 600/vara primera), Concepción (\$ 800/vara primera), Temuco (\$ 800/vara primera, \$100/vara segunda) y Valdivia (\$ 400/ vara primera, $\$ 100$ /vara segunda). El Cuadro 1 presenta la superficie dedicada al cultivo de calas, la producción de varas de primera y segunda selección y los ingresos correspondientes.

De acuerdo a la superficie de cultivo propuesta se consideró la adquisición de una parcela de $8.720 \mathrm{~m}^{2}$ colindante con la Ruta 5 Sur, con el propósito de rotar la zona de plantación anualmente. Se consideró un precio de adquisición de \$ 1.146 $\mathrm{m}^{2}$ (Fuente: Tattersal, Temuco). Las inversiones en infraestructura incluyeron bodega de insumos y materiales $\left(8 \mathrm{~m}^{2}\right)$, casa prefabricada para cuidador $\left(45 \mathrm{~m}^{2}\right)$, packing $\left(142,5 \mathrm{~m}^{2}\right)$ y oficina con baño $\left(10 \mathrm{~m}^{2}\right)$. Se contempló, además, la instalación de un cerco perimetral de $844 \mathrm{ml}$ y una torre de madera de $5 \mathrm{~m}$ de altura para contener un estanque de $3.000 \mathrm{~L}$ de agua. Las inversiones en equipamiento consideraron una cámara de refrigeración $\left(36 \mathrm{~m}^{2}\right)$, cámara de curado $\left(12,25 \mathrm{~m}^{2}\right)$, riego por cintas, bomba hidráulica, balanza electrónica de $5 \mathrm{~kg}$, bomba de espalda y estanque de almacenamiento de agua. Adicionalmente, se incluyó la adquisición de muebles y equipos de oficina; herramientas y ropa de trabajo; materiales de aseo, malla ruschell, mesones, postes, instalación eléctrica y la construcción de un pozo profundo. Se contempló para imprevistos un 5\% del valor total en inversiones.

Para la puesta en marcha del negocio se consideró la comisión que corresponde pagar a un corredor de propiedades por la compra del terreno, los gastos correspondientes a notaría, honorarios de un abogado, inscripción en el Conservador de Bienes Raíces e iniciación de actividades. El capital de trabajo se calculó mediante el Método del Déficit Acumulado Máximo (Sapag y Sapag, 1998) para el primer año de operación de la unidad productiva, considerando el pago al contado de todos los costos y la liquidación de las flores vendidas a 30 días, valor que incluye el IVA de la inversión inicial afecta a este impuesto. La depreciación de los activos fijos se realizó en forma lineal, considerando la vida útil de cada uno; la amortización de los activos nominales se realizó en 5 años y para el cálculo del valor residual se utilizó el método contable (Sapag y Sapag, 1998), excepto para el terreno que fue valorado a precio de mercado.

Dentro del personal necesario se consideró un administrador, un operario permanente y 2 operarios contratados entre octubre y abril para trabajos de campo y packing. Se incluyó el costo por visitas mensuales de un asesor en floricultura. Otros costos fijos correspondieron a consumo de electricidad, agua y teléfono; compra de útiles para oficina y aseo, contratación de la preparación de suelo y la mantención de los activos fijos, valor correspondiente anualmente al $1 \%$ de la inversión inicial. Se contempló un 10\% por imprevistos.

Los costos variables se agruparon en cinco ítemes: agroquímicos, fertilizantes, otros insumos agrícolas, embalaje y transporte. Dentro de los agroquímicos se consideró el barbecho químico (Gliphosato y Simazina), la desinfección de los bulbos (Captan y Benomil), el control de plagas y enfermedades (Benomil, Iprodione, Mancozeb, Pyrimethanil, Metamidofos, Endosulfuron, Lambdacihalotrina y Metaldehído). En fertilizantes se consideró la aplicación de salitre sódico $\left(90 \mathrm{~kg} \mathrm{ha}^{-1}\right)$, superfosfato triple (306 kg ha-1), muriato de potasio $\left(22 \mathrm{~kg} \mathrm{ha}^{-1}\right)$,

Cuadro 1

Ingreso anual (miles \$) correspondiente a la producción calas de color en la Región de La Araucanía, Chile

\begin{tabular}{|c|c|c|c|c|r|c|}
\hline \multirow{2}{*}{ Año } & \multicolumn{2}{|c|}{ Cantidad de varas } & \multirow{2}{*}{$\begin{array}{c}\text { Superficie } \\
\left(\mathbf{m}^{2}\right)\end{array}$} & \multicolumn{2}{|c|}{ Venta de calas (M\$) } & \multirow{2}{*}{$\begin{array}{c}\text { Ingresos } \\
\end{array}$} \\
\cline { 2 - 2 } & Primera & Segunda & & Primera & Segunda & Totales (M\$) \\
\hline \multirow{2}{*}{1} & 31.639 & 7.910 & 1.055 & $20.249,0$ & 791,0 & $21.039,9$ \\
2 & 34.803 & 8.701 & 1.160 & $22.273,9$ & 870,1 & $23.143,9$ \\
3 & 38.283 & 9.571 & 1.276 & $24.501,2$ & 957,1 & $25.458,3$ \\
4 & 42.112 & 10.528 & 1.404 & $26.951,4$ & $1.052,8$ & $28.004,2$ \\
$5-10$ & 46.323 & 11.581 & 1.544 & $29.646,5$ & $1.158,1$ & $30.804,6$ \\
\hline
\end{tabular}


boronato de calcita $\left(12 \mathrm{~kg} \mathrm{ha}^{-1}\right)$, fertiyeso $(236 \mathrm{~kg}$ $\mathrm{ha}^{-1}$ ) y los fertilizantes foliares Urexal cal y Nitrofosca ( $2 \mathrm{~L} \mathrm{ha}^{-1}$ cada uno). Otros insumos correspondieron a humectante, reguladores del crecimiento y viruta de pino para mantener la humedad del suelo y controlar malezas en la platabanda. El embalaje consistió en cajas de cartón y el transporte consideró el traslado de la producción de calas por bus hasta las ciudades de Santiago, Concepción y Valdivia y en camioneta en la ciudad de Temuco.

Como criterios de evaluación se utilizó el Valor Actual Neto (VAN) y la Tasa Interna de Retorno (TIR). El primero plantea que el proyecto debe aceptarse si su VAN es igual o superior a cero, mientras que si la TIR es igual o mayor que la tasa de descuento utilizada para calcular el VAN, el proyecto se acepta y si es menor se rechaza (Sapag y Sapag, 1998). Matemáticamente se expresan de la siguiente forma:

$$
V A N=\sum \frac{F_{t}}{(1+i)^{t}}-I_{0} \quad \sum \frac{F_{t}}{(1+r)^{t}}-I_{0}=0
$$

donde Ft es el flujo neto de caja del periodo $t, I_{\mathrm{o}}$ es la inversión inicial, $i$ corresponde a la tasa de descuento o de costo de capital, y $r$ corresponde a la tasa interna de retorno.

Para el caso del cálculo del VAN, considerando que el 100\% de la inversión es financiada con aportes propios, se utilizó una tasa de descuento del $12 \%$. La tasa de descuento para evaluar la rentabilidad de la inversión financiada con deuda se calculó utilizando la fórmula correspondiente al Costo Promedio Ponderado de Capital (Ross, Westerfield y Jaffe, 1997), el cual pondera el costo del capital patrimonial y el costo de la deuda después de impuestos y se expresa de la siguiente forma:

\section{Costo Promedio Ponderado de Capital =}

$$
\left(\frac{S}{S+B}\right) r_{s}+\left(\frac{B}{S+B}\right) r_{B}\left(1-T_{c}\right)
$$

Donde $S$ corresponde a la fracción del financiamiento vía patrimonio, $B$ es la parte cubierta a través de endeudamiento, $r_{\mathrm{S}}$ es el costo del capital, $r_{\mathrm{B}}$ corresponde al costo de la deuda y $T c$ es la tasa de impuesto a las utilidades corporativas, que correspondió a $17 \%$. Como tasa de costo del capital se utilizó $12 \%$ y como costo de la deuda una tasa del
$8 \%$, considerando un crédito a 10 años para la inversión inicial. La tasa de descuento correspondiente a una estructura de capital con $50 \%$ de deuda correspondió a 9,3\%. Se consideró además un segundo crédito a 6 años para la reinversión en material vegetal correspondiente al año 4 y a 2 años para la reinversión en túberos al octavo año de evaluación.

Además, se calculó el período de recuperación de la inversión y el perfil del VAN. El primero definido como el tiempo que se requiere para que los capitales invertidos se igualen con los flujos netos de ingresos generados por la inversión, mientras que el segundo permite apreciar globalmente la tendencia que sigue el VAN frente a diferentes tasas de descuento. Finalmente, se incluye un análisis de sensibilidad, considerando el efecto de variaciones en los precios de venta y en el volumen de producción para ambas estructuras de capital.

\section{RESULTADOS Y DISCUSIÓN}

El Cuadro 2 presenta los valores correspondientes a la inversión total en activos fijos, nominales y capital de trabajo necesarios para la implementación de la producción de calas de color. Los activos fijos representan el 83,4\% de la inversión total, los activos nominales el $0,4 \%$ y el capital de trabajo cerca el 16,4\%. El principal ítem de inversión corresponde a la compra de los túberos de cala que representan el $44,2 \%$ del activo fijo, seguido por las obras civiles $(23,3 \%)$, el terreno $(11,6 \%)$ y maquinarias y equipos $(9,5 \%)$.

Dentro del costo fijo anual (Cuadro 2), el de mayor relevancia corresponde al pago de sueldos del personal que en conjunto representa el 71,4\% del total, seguido por los imprevistos considerados, la asesoría externa (7,3\%), mantención de los activos fijos $(5,6 \%)$ y el pago por los servicios de electricidad y agua. El Cuadro 3 presenta el resumen de costos variables, destacando que los de mayor relevancia corresponden a costos de comercialización. El ítem de mayor importancia, embalaje de las flores, tiene una participación anual aproximada entre 35 y $50 \%$, mientras que en segundo lugar se encuentra el transporte hasta las florerías que representa anualmente entre 37 y $48 \%$, aproximadamente. Dentro de los costos variables de producción el de mayor cuantía corresponde al uso de agroquímicos, dentro de los cuales destacan fertilizantes e insecticidas. 
Cuadro 2

Inversiones en activos fijos, activos nominales y capital de trabajo y costos fijos (valores netos miles \$) correspondientes a la producción calas de color en la Región de La Araucanía, Chile

\begin{tabular}{|c|c|}
\hline Inversiones año 0 & M\$ \\
\hline Terreno & $10.000,0$ \\
\hline Obras civiles & $20.160,0$ \\
\hline Máquinas y equipos & $8.181,6$ \\
\hline Muebles y útiles & 690,8 \\
\hline Herramientas & 593,0 \\
\hline Instalación eléctrica & $3.281,4$ \\
\hline Material vegetal & $38.270,0$ \\
\hline Otros activos fijos & $3.524,5$ \\
\hline Imprevistos $(5 \%)$ & $1.822,2$ \\
\hline Total activos fijos & $86.523,5$ \\
\hline Valor residual de los activos & $22.096,0$ \\
\hline Comisión corredor & 100,0 \\
\hline Notaría & 50,0 \\
\hline Abogado & 200,0 \\
\hline Inscripción conservador bienes raíces & 50,0 \\
\hline Total activos nominales & 400,0 \\
\hline Capital de trabajo & $16.949,2$ \\
\hline IVA inversión inicial & $28.534,3$ \\
\hline Inversión total & 103.472,7 \\
\hline \multicolumn{2}{|l|}{ Costos fijos } \\
\hline Sueldo ingeniero agrónomo & $7.200,0$ \\
\hline Operario permanente & $1.440,0$ \\
\hline Mano de obra temporal & $1.200,0$ \\
\hline Asesoría especializada en floricultura & $1.000,0$ \\
\hline Consumo telefónico & 180,0 \\
\hline Útiles varios & 96,0 \\
\hline Mantención activos fijos & 765,2 \\
\hline Servicios (agua, energía eléctrica) & 621,8 \\
\hline Preparación de suelo & 19,0 \\
\hline Imprevistos ( $10 \%$ costos fijos) & $1.252,2$ \\
\hline Total costos fijos & $13.774,3$ \\
\hline
\end{tabular}

Al considerar el costo total (fijos + variables), destaca la importancia del costo fijo que representa anualmente proporciones que fluctúan entre 93 y $95 \%$. Considerando esta estructura de costos y la producción anual de varas comercializables de primera y segunda selección, el costo total unitario fluctúa entre \$ 305 y \$371/vara los tres primeros años, alcanza a \$278/vara el cuarto año y varía entre \$ 253 y \$256/vara a partir del quinto año de producción. Estos resultados indican que el precio de mercado de las calas de segunda selección (\$ 100/unidad en Temuco y Valdivia) es menor al costo unitario por vara, con una diferencia negativa que fluctúa entre \$ 187 y \$271/vara.

El Cuadro 4 presenta los resultados de la evaluación económica considerando las estructuras de capital propuestas. Como se observa, en ambos casos las cifras obtenidas para el VAN son negativas, lo que implica que la producción y comercialización de calas de color genera pérdidas en las condiciones propuestas y bajo las rentabilidades exigidas. Destaca la menor pérdida correspondiente a la estructura de capital con deuda debido a que se mide sólo la rentabilidad de la inversión de los recursos propios. Respecto a la rentabilidad esperada, debido a que bajo ambas estructuras de capital se producen pérdidas operacionales (años 4 y 8 , considerando $100 \%$ de aporte propio; años 1, 4, 8 y 9 con estructura de capital con deuda) asociadas principalmente a las reinversiones en material vegetal, no fue posible calcular la TIR como originalmente se propuso, debiéndose recurrir al cálculo de la Tasa Interna de Retorno Modificada (TIRM) que considera que los flujos de caja se reinvierten a una tasa distinta de la TIR (10\%), y así se evita la obtención de TIR múltiples. En base a esto se obtuvieron rentabilidades de $3,1 \%$ al financiar las inversiones en su totalidad con aportes propios y negativa al incluir crédito en la estructura de financiamiento. Además, los flujos de caja correspondientes a los años 1 y 9 no alcanzan a cubrir las cuotas crediticias necesarias para cancelar la deuda considerada en la estructura de capital con 50\% de aporte propio, lo que explica que la TIRM sea negativa. A esto se debe agregar que el período de recuperación de la inversión es superior a 10 años, lo que implica la imposibilidad de recuperar la inversión inicial en el período evaluado.

En el mismo cuadro se presentan los resultados del análisis de sensibilidad, el cual, dados los valores de VAN obtenidos, pretende determinar las condiciones en que el negocio no generaría pérdidas o punto de quiebre (Sapag y Sapag, 1998). Como se observa, para no generar pérdidas se requiere un menor incremento en los precios de venta de las flores o en el volumen de ventas al financiar la inversión en parte a través de un crédito. El VAN toma el valor cero frente a un incremento de $17,8 \%$ en los precios (Valdivia: \$ 471/vara prime- 
Cuadro 3

Costos variables totales (valores netos miles \$) correspondientes a la producción calas de color en la Región de La Araucanía, Chile

\begin{tabular}{|c|c|c|c|c|c|c|}
\hline Año & Agroquímicos & Fertilizantes & Otros insumos & Embalaje & Transporte & Totales \\
\hline 1 & 156,5 & 67,3 & 25,1 & 305,2 & 328,6 & $\mathbf{8 8 2 , 7}$ \\
2 & 13,3 & 15,8 & 20,8 & 338,8 & 339,9 & $\mathbf{7 2 8 , 6}$ \\
3 & 20,2 & 28,6 & 25,4 & 371,5 & 352,4 & $\mathbf{7 9 8 , 1}$ \\
4 & 46,6 & 23,8 & 25,6 & 410,4 & 366,1 & $\mathbf{8 7 2 , 6}$ \\
5 & 121,9 & 43,2 & 25,7 & 448,5 & 381,3 & $\mathbf{1 . 0 2 0 , 7}$ \\
6 & 21,5 & 42,4 & 25,7 & 448,5 & 381,3 & $\mathbf{9 1 9 , 4}$ \\
7 & 24 & 33,9 & 25,7 & 448,5 & 381,3 & $\mathbf{9 1 3 , 4}$ \\
8 & 137,6 & 41,2 & 25,7 & 448,5 & 381,3 & $\mathbf{1 . 0 3 4 , 3}$ \\
9 & 27,1 & 33,9 & 25,7 & 448,5 & 381,3 & $\mathbf{9 1 6 , 4}$ \\
10 & 17,2 & 29,1 & 25,7 & 448,5 & 381,3 & $\mathbf{9 0 1 , 8}$ \\
\hline
\end{tabular}

\section{Cuadro 4}

Indicadores económicos y resultados del análisis de sensibilidad correspondientes a la producción calas de color en la Región de La Araucanía, Chile

\begin{tabular}{|c|c|c|}
\hline Indicadores & Financiamiento & A \\
\hline VAN (M\$) & $\begin{array}{c}100 \% \text { propio } \\
50 \% \text { propio/50\% deuda }\end{array}$ & $\begin{array}{l}-70.138 \\
-41.472\end{array}$ \\
\hline $\operatorname{TIRM}(\%)$ & $\begin{array}{c}100 \% \text { propio } \\
50 \% \text { propio/50\% deuda }\end{array}$ & $\begin{array}{r}3,1 \\
-2,3\end{array}$ \\
\hline \multicolumn{2}{|c|}{ Período de recuperación de la inversión } & $>10$ años \\
\hline \multicolumn{3}{|c|}{ Variación porcentual en el precio de venta para alcanzar el punto de equilibrio del VAN } \\
\hline $\begin{array}{l}100 \% \text { propio } \\
50 \% \text { propio/50\% deuda }\end{array}$ & & $\begin{array}{c}55,01 \% \\
17,8 \%\end{array}$ \\
\hline \multicolumn{3}{|c|}{ Variación porcentual en el volumen de venta para alcanzar el punto de equilibrio del VAN } \\
\hline $\begin{array}{l}100 \% \text { propio } \\
50 \% \text { propio } / 50 \% \text { deuda }\end{array}$ & & $\begin{array}{l}55,3 \% \\
17,9 \%\end{array}$ \\
\hline
\end{tabular}

ra calidad, \$118/vara segunda calidad; Temuco: \$ 942/vara primera calidad, \$ 118/vara segunda calidad; Concepción: \$942/vara; Santiago: \$ 707/ vara), siendo necesarios precios aproximadamente $30 \%$ superiores para evitar pérdidas al financiar la inversión en forma total con recursos propios (Valdivia: \$ 620/vara primera calidad, \$ 155/vara segunda calidad; Temuco: \$ 1.240/vara primera calidad, \$155/vara segunda calidad; Concepción:
\$ 1.240/vara; Santiago: \$ 930/vara). Respecto a la sensibilización de los volúmenes de producción, el punto de quiebre se obtiene al comenzar la producción con $1.635 \mathrm{~m}^{2}$ y alcanzar un régimen de $2.393 \mathrm{~m}^{2}$ en el caso de financiar la inversión exclusivamente con aportes propios, mientras que al considerar financiamiento de parte de la inversión vía crédito es necesario comenzar con $1.243 \mathrm{~m}^{2} \mathrm{y}$ llegar a cultivar $1.820 \mathrm{~m}^{2}$, a partir del quinto año 
de evaluación. Esto evidencia un efecto positivo asociado a la existencia de apalancamiento (leveraje) operativo, que se origina en que a una mayor producción los costos fijos por unidad disminuyen (Ross et al., 1997). A la vez, el menor incremento del volumen de producción para evitar pérdidas al incluir deuda en la estructura de capital se explica por efecto de la existencia de apalancamiento financiero generado por el uso deuda, puesto que a mayor número de unidades vendidas la carga financiera estará mejor distribuida, lográndose un menor costo financiero por unidad (Ross et al., 1997).

Los resultados del perfil del VAN (Figura 1) indican que la producción y comercialización de calas de color no resulta rentable frente a ninguna tasa de rentabilidad exigida, bajo las condiciones

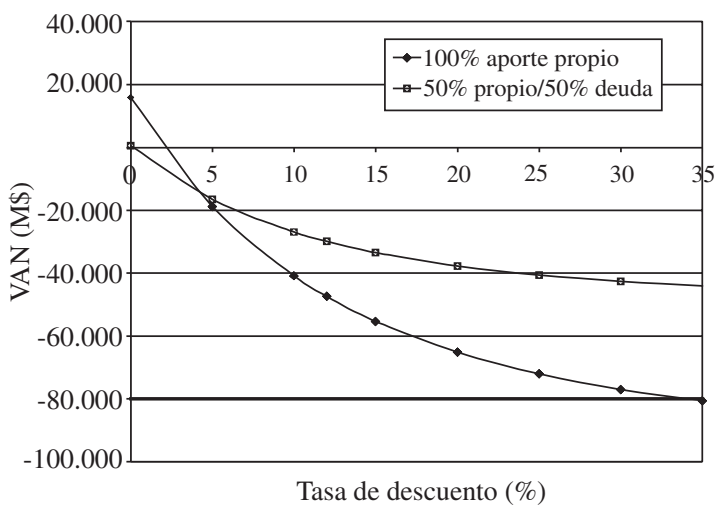

Figura 1. Perfil del VAN de la comercialización de calas de color producidas en la Región de La Araucanía-Chile, con diferentes formas de financiamiento de la inversión.

\section{LITERATURA CITADA}

CLEMENS, J., DENNIS, D., BUTLER, R., THOMAS, M., INGLE, A. AND WELSH, T. 1998. Mineral nutrition of Zantedeschia plants affects plant survival, tuber yield, and flowering upon replanting. Journal of Horticultural Sciences \& Biotechnology 73(6): 755-762.

CORTÉS-MONLLOR, A. 1990. Erwinia carotovora pv carotovora, causal agent of soft rot disease in some crop in Puerto Rico. J. Agric. Univ. P. R. 74(1): 83-92.

CHAIN, M. 2000. El cultivo de calas. Informativo INIA Carillanca $\mathrm{N}^{\mathrm{o}} 7$.

CHEN, J., LIU, M. AND HO, Y. 2000. Size on in vitro plantlets affects subsequent tuber production of acclimatized cala lily. Hortscience 35(2): 290-292.

ETCHEVERRÍA, P. 2002. Efecto de la densidad de sombra y del mulch en la producción y calidad de flores y túberos de técnicas y de mercado consideradas si la inversión es financiada en su totalidad con recursos propios, mientras que si es financiada en un $50 \%$ con deuda el negocio es rentable a tasas iguales o inferiores al $2 \%$. Este resultado se relaciona en gran medida con el monto de la inversión inicial y reinversiones requeridas para renovar el material vegetal, según propone la tecnología de producción utilizada. Es así como al eliminar las reinversiones en túberos correspondientes a los años 4 y 8 de la evaluación se reducen las pérdidas, obteniéndose un VAN de M\$-29.463 y M\$-14.449 al financiar sin y con deuda, respectivamente. Esto indica claramente que la limitación técnica del negocio evaluado radica en la posibilidad de producir en forma interna los túberos de cala necesarios para sustentar los volúmenes de producción y venta propuestos o utilizar otra forma de propagación. Al respecto, Chen et al. (2000) obtuvieron mejores resultados productivos en calas obtenidas mediante micropropagación, respecto de plantas obtenidas a partir de túberos.

No obstante, es necesario señalar en forma paralela la existencia de limitaciones de mercado, por cuanto, aun cuando no fueran necesarias las reinversiones en material vegetal, se requiere un aumento superior a 23 y $11 \%$ al financiar las inversiones con recursos propios o a través de crédito, respectivamente, en los precios o en los volúmenes de venta en forma independiente, para generar utilidades sobre la inversión. Esto se relaciona con los bajos precios pagados por las flores de segunda selección que representan el 20\% de las varas comercializadas, precios que son inferiores al costo unitario total.
Zantedeschia hybrida cv. Mango. Tesis Ingeniero Agrónomo, Universidad de La Frontera. 67 p.

FUNNELL, K., TJIA, B., STANLEY, C., COHEN, D. AND SEDCOLE, J. 1988. Effect of storage temperature, duration and gibberellic acid on the flowering of Zantesechia elliottiana ans Z. "Pink Satin". J. Amer. Soc. Hort. Sci. 113: 860-863.

FUNNELL, K., HEWETT, E., WARRINGTON, I. AND PUMMER, J. 1998. Leaf mass portioning as a determinant of dry matter accumulation in Zantedeschia. Journal American Society Horticultural Science 123 (6): 973-979.

KRITZINGER, E., JANSEN VAN VUUREN, R., WOODWARD, B., RONG, I., SPREETH, M. AND SLABBERT, M. 1998. Elimination of external and internal contaminants 
in rhizomes of Zantedeschia aethiopica with commercial fungicides and antibiotics. Plant Cell, Tissue and Organ Culture 52: 61-65.

MERA, A. 2004. Estudio de prefactibilidad técnico-económica para la comercialización de calas de colores, producidas en la IX Región. Tesis Ingeniero Agrónomo, Universidad de La Frontera. 114 p.

ODEPA, 2005. Flores de corte. Disponible en: http:// www.odepa.cl. Conectado el 11 de octubre de 2005.
ROSS, R., R. WESTERFIELD, Y J. JAFFE. 1997. Finanzas corporativas. $3^{\mathrm{a}}$ ed. Mc Graw-Hill/Interamericana, Madrid, España. 984 p.

SAPAG, N., Y R. SAPAG. 1998. Preparación y evaluación de proyectos: Criterios de evaluación de proyectos. Mirtha Edna Suárez. $3^{\mathrm{a}}$ ed. McGraw-Hill, Santa Fe de Bogotá, D.C., Colombia. 403 p.

TJIA, B. 1987. Growth regulator effect on growth and flowering of Zantedeschia rehmannii hib. Hortscience 22(3): 507-508. 